\title{
The role of CD133 expression in the carcinogenesis and prognosis of patients with lung cancer
}

\author{
HANBO LE ${ }^{1}$, FANG ZENG ${ }^{2}$, LIYUN XU ${ }^{2}$, XIAOGUANG LIU ${ }^{2 *}$ and YANYAN HUANG ${ }^{2 *}$ \\ ${ }^{1}$ Department of Cardio-Thoracic Surgery, Zhoushan Hospital of Zhejiang Province; ${ }^{2}$ Joint Laboratory of Immunogenomics, \\ Zhoushan Hospital-BIG/CAS, Zhoushan, Zhejiang 316004, P.R. China
}

Received March 8, 2013; Accepted August 28, 2013

DOI: $10.3892 / \mathrm{mmr} .2013 .1667$

\begin{abstract}
Cancer stem cells (CSCs) are a small population of undifferentiated cancer cells within tumors, which contribute to tumorigenicity and relapse. In the current study, CD133 (also termed prominin-1), a CSC marker, was investigated to determine its involvement in predicting carcinogenesis and prognosis in patients with non-small cell lung carcinoma (NSCLC). CD133-positive lung cancer cells were isolated to analyze self-renewal, differentiation and tumorigenic abilities in vitro and in vivo. Quantitative polymerase chain reaction was used to detect the expression of CD133 and three other CSC-associated markers, octamer-binding transcription factor 4 (OCT4A), Nanog homeobox (NANOG) and multidrug resistance protein 1 (MDR1), in primary NSCLC and adjacent non-cancer tissues. A series of statistical methods were used to analyze the correlation between mRNA expression levels, clinicopathological features and patient survival. The results showed that CD133-positive NSCLC cells demonstrated clonogenic, tumorigenic and drug-resistance properties compared with their CD133-negative counterparts or parental cells. In addition, compared with the adjacent normal lung tissue, the levels of CSC-associated biomarkers CD133, OCT4A, NANOG and MDR1 were significantly increased in NSCLC tissue. Elevated expression of CD133 was associated with stage, tumor size and differentiation of NSCLC; however, the cox hazard regression analysis showed no significant association between CD133 expression and overall patient survival. The present study supports the hypothesis that the stem cell population can be enriched in cells expressing the CD133 cell
\end{abstract}

Correspondence to: Professor Xiaoguang Liu or Dr Yanyan Huang, Joint Laboratory of Immunogenomics, Zhoushan Hospital-BIG/CAS, Zhoushan, Zhejiang 316004, P.R. China

E-mail:1xg2004@gmail.com

E-mail: 2005huangyanyan@163.com

*Contributed equally

Key words: non-small cell lung carcinoma cells, cancer stem cells, CD133, flow cytometry, xenograft, quantitative polymerase chain reaction, prognosis surface marker and that highly expressed CD133 is involved in the occurrence of NSCLC. However, CD133 may not be considered as an independent factor in predicting the prognosis of patients with NSCLC. Further studies are required to investigate the association between CD133 expression and overall patient survival.

\section{Introduction}

Lung cancer is the most common cause of cancer-related mortality worldwide, and a poor five-year survival rate $(15 \%)$ highlights the importance of gaining an improved understanding of this malignancy to improve prevention, diagnosis and treatment (1). A previous study showed that tumor initiation and propagation is induced by a specific population of self-renewing tumor cells, termed cancer stem cells (CSCs) or tumor propagating cells (2). Previous studies have indicated that similar to a number of solid tumors, human lung cancers, particularly non-small cell lung carcinoma (NSCLC), harbor CSC populations (3-5).

Certain molecules are being investigated as putative markers of CSCs in malignancies, including lung cancer (6). CD133 (also termed prominin-1), a cell-surface glycoprotein comprising five transmembrane domains and two large glycosylated extracellular loops, has been previously used as a biomarker to separate cancer stem cells from a variety of solid human tumors, including brain (7), breast (8), liver (9), pancreas (10), colon (11) and lung $(12,13)$ tumors. Although CD133 has been used to enrich lung cancer CSCs in several studies, the potential for the use of CD133 as a key marker remains unclear (14-16). In addition, the use of CD133 as a prognostic marker in lung cancer has not yet been confirmed due to conflicting studies (17-22). To investigate these divergent observations, the involvement of CD133 was analyzed using NSCLC cells and clinical specimens to evaluate a possible correlation between CD133 expression and clinical-pathological variables in patients with NSCLC. To the best of our knowledge, this was the first study to investigate NSCLC tumor and non-tumor tissue, with respect to CSC-marker expression profiles and their relevance to the clinicopathological parameters of lung cancer. The current results demonstrated that elevated expression of CD133 was associated with early clinical stages, larger tumor size and poor differentiation of NSCLC. 


\section{Materials and methods}

Cell lines and isolation of CD133-positive cells. NSCLC cells, A549, H1299, SPC-A1, SK-MES-1 and a human bronchial epithelial cell line (HBE), were cultured separately in RPMI-1640 medium (Gibco, Invitrogen Life Technologies, Carlsbad, CA, USA) supplemented with $10 \%$ fetal bovine serum (FBS), $100 \mathrm{IU} / \mathrm{ml}$ penicillin and $100 \mathrm{mg} / \mathrm{ml}$ streptomycin in a $5 \% \mathrm{CO}_{2}$ humidified atmosphere at $37^{\circ} \mathrm{C}$. For isolation of CD133-positive cells, the parental H1299 cells were labeled with $1 \mathrm{ml} \mathrm{CD133}$ per liter micromagnetic beads per million cells, using a CD133 cell isolation kit (CD133 MicroBead kit; Miltenyi Biotec, Auburn, CA, USA). The isolated CD133-positive cells were cultured in medium consisting of serum-free Dulbecco's modified Eagle's medium (DMEM)/F-12 medium (Gibco, Invitrogen Life Technologies) supplemented with $20 \mathrm{ng} / \mathrm{ml}$ human epidermal growth factor (EGF) (PeproTech, Rocky Hill, NJ, USA), 10 ng/ml human basic fibroblast growth factor (bFGF; PeproTech) and 2\% B-27 serum-free supplements (Invitrogen Life Technologies). The medium was replaced or supplemented with fresh growth factors twice weekly until floating aggregates formed.

To determine the percentage of single cells capable of regenerating novel spheres, cells were plated at a density of 1,000 cells/ml in ultralow-attachment 6-well plates (Corning Life Sciences, Union City, CA, USA) to obtain the novel spheres. The total number of tumor spheres was counted following 10 days of culture. Efficiency of sphere formation was calculated by the following equation: (Total number of spheres formed / total number of living cells seeded) x 100 .

Patients and tissue samples. NSCLC and matched adjacent normal biopsies were obtained from 30 patients (see supplementary Table I) undergoing pulmonary resection at the Zhoushan Hospital of Zhejiang province (Zhoushan, Zhejiang, China) between January 2009 and May 2010. All samples were collected with informed consent obtained at an internal review. The ethics board committees of the Zhoushan Hospital of Zhejiang province approved the current study. Patients recruited into the study had not undergone chemotherapy or radiotherapy prior to surgery. Surgical specimens of the resected tumors were collected following the confirmation of diagnosis by pathological examination. Tumor sections and matched adjacent noncancerous tissues, were placed in separated cryovials and snap-frozen in liquid nitrogen until analysis. All cases were reviewed by two pathologists and diagnosis was confirmed according to criteria previously established by the National Comprehensive Cancer Network (23).

Flow cytometry and fluorescence-activated cell sorting (FACS) analyses. For flow cytometry analysis, cells $\left(10^{6} / 100 \mu 1\right)$ were dissociated using non-enzymatic solution Cellstripper (Mediatech, Herndon, VA, USA) and incubated with the appropriate dilution of control or specific Phycoerythrin (PE)-conjugated anti-CD133/2 antibody (Miltenyi Biotec) at $4^{\circ} \mathrm{C}$ for $10 \mathrm{~min}$. All samples were measured using a FACSCalibur flow cytometer (BD Biosciences, Franklin Lakes, NJ, USA) and analyzed with CellQuest software (BD Biosciences).
RNA extraction and quantitative polymerase chain reaction $(q P C R)$. Total RNA from specimens or cells was extracted using TRIzol (Invitrogen Life Technologies), according to the manufacturer's instructions and the concentration was determined using a Bioanalyzer UV spectrophotometer Q3000 (Quawell Technology, Inc., San Jose, CA, USA). Complementary DNA (cDNA) was reverse transcribed by $3 \mu \mathrm{g}$ total RNA in a $20 \mu \mathrm{l}$ reaction using $0.5 \mu \mathrm{g}$ Oligo(dT) $)_{18}$ primer and 200 units of RevertAid ${ }^{\mathrm{TM}}$ M-MuLV Reverse Transcriptase (Fermentas, Burlington, ON, Canada) and treated with RNase-Free DNase (Qiagen, Valencia, CA, USA). The primers of the housekeeping gene glyceraldehyde-3-phosphate dehydrogenase (GAPDH) and target genes CD133, octamer-binding transcription factor 4 (OCT4A), sex determining region Y-box 2 (SOX2) and Nanog homeobox (NANOG), multidrug resistance protein 1 (MDR1) and ATP-binding cassette subfamily G member 2 (ABCG2) are shown in Table I. The cDNA amplification was performed in a final reaction volume of $20 \mu \mathrm{l}$ containing $0.5 \mu \mathrm{M}$ concentrations of each primer, $8 \mu 12.5 \mathrm{X}$ Real Master mix, $1 \mu 120 \mathrm{X}$ SYBR solution (Tiangen Biotech, Shanghai, China) and $2 \mu \mathrm{l}$ 1:10 diluted cDNA. PCR was prepared in triplicate and heated to $95^{\circ} \mathrm{C}$ for $10 \mathrm{~min}$, followed by 40 cycles of the following sequences: Denaturation at $95^{\circ} \mathrm{C}$ for $15 \mathrm{sec}$, annealing at $60^{\circ} \mathrm{C}$ for $20 \mathrm{sec}$ and extension at $68^{\circ} \mathrm{C}$ for $35 \mathrm{sec}$. The expression of genes was detected by qPCR using Applied Biosystems 7500 Real-Time RT-PCR system (Applied Biosystems, Carlsbad, $\mathrm{CA}$, USA). The cycle threshold $(\mathrm{Ct})$ values were calculated with the SDS 2.0.1 software (Applied Biosystems). All target gene $\mathrm{Ct}$ values were determined in reference to GAPDH using the $2^{-\Delta \Delta C t}$ method (24).

Chemotherapy resistance studies. Parental H1299 cells and sorted cells $\left(5 \times 10^{3}\right)$ were plated in 96-well plates. Cisplatin and paclitaxel (Sigma-Aldrich, St. Louis, MO, USA) were added to the culture medium at a final concentration of $25 \mu \mathrm{g} / \mathrm{ml}$ and $20 \mu \mathrm{M}$, respectively, for $24 \mathrm{~h}$. Subsequently, cell viability was analyzed by an MTT assay.

In vivo analysis of tumor growth. Female nude mice (strain, BALB/c; age, 4 weeks) were obtained from the Shanghai Laboratory Animal Center (Shanghai, China). Specific numbers of parental and sorted cells $\left(10^{3}, 10^{4}\right.$ or $10^{6}$ cells) were subcutaneously injected into the flank of the nude mice. Tumor growth was monitored and volume was measured weekly using a caliper (volume $=$ width $\mathrm{x}$ length $^{2} \mathrm{x} \pi / 6$ ) for a maximum of 8 weeks. Subsequently, mice were euthanized by cervical vertebra dislocation and the tumors were collected, fixed in formalin and embedded in paraffin for further use.

Statistical analysis. Statistical analyses were performed with the GraphPad Prism 5.0 statistical software (GraphPad Software, Inc., La Jolla, CA, USA). The paired t-test, unpaired t-test and Mann-Whitney U test were used to analyze the correlation between mRNA expression levels and the clinicopathological features. The paired sample t-test was used to compare the differences of mRNA expression between lung tumors and the surrounding normal tissue. Survival analysis was estimated by the Kaplan-Meier method and the log-rank test was used to compare the survival between groups. The 
Table I. Primer sequences of CSC-associated genes for qPCR.

\begin{tabular}{|c|c|c|c|}
\hline Gene (accession no.) & Primers sequence (5'-3') & Product size (bp) & $\operatorname{Tm}\left({ }^{\circ} \mathrm{C}\right)$ \\
\hline OCT4A (NM_002701) & $\begin{array}{l}\text { F: GTGGAGAGCAACTCCGATG } \\
\text { R: TGCTCCAGCTTCTCCTTCTC }\end{array}$ & 86 & 60 \\
\hline SOX2 (NM_003106) & $\begin{array}{l}\text { F: CGAGTGGAAACTTTTGTCGGA } \\
\text { R: TGTGCAGCGCTCGCAG }\end{array}$ & 74 & 60 \\
\hline NANOG (NM_024865) & $\begin{array}{l}\text { F: ATTCAGGACAGCCCTGATTCTTC } \\
\text { R: TTTTTGCGACACTCTTCTCTGC }\end{array}$ & 76 & 60 \\
\hline MDR1 (NM_000927) & $\begin{array}{l}\text { F: TGGCAAAGAAATAAAGCGACTGA } \\
\text { R: CAGGATGGGCTCCTGGG }\end{array}$ & 76 & 60 \\
\hline ABCG2 (NM_004827) & $\begin{array}{l}\text { F: CCCCAGGCCTCTATAGCTCAGATCA } \\
\text { R: TCCACGGCTGAAACACTGCTGA }\end{array}$ & 164 & 60 \\
\hline CD133 (NM_006017) & $\begin{array}{l}\text { F: CACTACCAAGGACAAGGCGT } \\
\text { R: TCCTTGATCGCTGTTGCCAT }\end{array}$ & 134 & 60 \\
\hline GAPDH (NM_002046) & $\begin{array}{l}\text { F: CATCATCCCTGCCTCTACTG } \\
\text { R: GCCTGCTTCACCACCTTC }\end{array}$ & 180 & 60 \\
\hline
\end{tabular}

CSC, cancer stem cell; qPCR, quantitative polymerase chain reaction; Tm, melting temperature; bp, base pair; OCT4A, octamer-binding transcription factor 4; F, forward; R, reverse; SOX2, sex determining region Y-box 2; NANOG, Nanog homeobox; MDR1, multidrug resistance protein 1; ABCG2, ATP-binding cassette subfamily G, member 2; GAPDH, glyceraldehyde 3-phosphate dehydrogenase.

Cox proportional hazard regression model was used to analyze the risk factors for lung cancer. $\mathrm{P}<0.05$ was considered to indicate a statistically significant difference.

\section{Results}

Isolation and characterization of CD133-positive cells from lung cancer cell lines. To investigate the involvement of CD133 in the link between CSCs and lung cancer biology, CD133-positive cells were isolated from lung cancer cell lines using the magnetic bead method to determine the expression levels of CD133. CD133 was shown to be highly expressed in, 95C, SPC-A1, A549 and H1299 NSCLC lines. The highest expression levels were observed in the H1299 cells (Fig. 1A). Thus, H1299 cells were used for all subsequent experiments.

The isolated CD133-positive cells identified by flow cytometry formed floating sphere-like bodies in DMEM/ F-12 serum-free medium with bFGF and EGF. When CD133-positive cells were grown under adherent conditions in culture medium supplemented with $10 \% \mathrm{FBS}$, the cells acquired the typical morphological features of parental H1299 cells with the loss of CD133 expression (decrease in expression from 94.9 to $4.9 \%$; Fig. 1B). qPCR results demonstrated that the expression of stemness genes (OCT4A, SOX2 and NANOG) and drug resistant genes (MDR1 and ABCG2) in CD133-positive cells were higher compared with those in the CD133-negative cells (Fig. 1C). The ability to form spheroid-like bodies was significantly higher in the CD133-positive cells compared with the parental or CD133-negative cells $(\mathrm{P}<0.05$; Fig. 1D). The multidrug chemotherapy resistant abilities of the CD133-positive cells and CD133-negative cells were then investigated. Compared with the CD133-negative and parental cells, CD133-positive cells sorted from H1299 cells were more resistant to cisplatin and paclitaxel $(\mathrm{P}<0.05$; Fig. 1E).
Table II. Tumor formation derived from parental and sorted H1299 cells.

\begin{tabular}{lccc}
\hline Cells & $10^{3}$ cells & $10^{5}$ cells & $10^{6}$ cells \\
\hline Parental & $0 / 4$ & $1 / 4$ & $3 / 4$ \\
CD133-positive & $2 / 4$ & $4 / 4$ & $4 / 4$ \\
CD133-negative & $0 / 4$ & $0 / 4$ & $1 / 4$ \\
\hline
\end{tabular}

Specific number $\left(10^{3}, 10^{4}\right.$ and $10^{6}$ cells $)$ of the parental and sorted cells H1299 cells were subcutaneously injected into the flanks of nude mice for a maximum of 8 weeks. Tumor formation was analyzed two months following implantation. Four mice per group.

To analyze the tumorigenic potential of CD133-positive cells grown under sphere-forming conditions, during which this property of stem cells may be maintained, specific numbers of H1299 CD133-positive, CD133-negative and parental cells were subcutaneously implanted into the flanks of nude mice (BALB/c strain). As shown in Table II, tumor growth was observed in all groups in which mice were inoculated with $10^{3}-10^{6}$ CD133-positive cells, whereas no tumor growth was observed following inoculation with $10^{3} \mathrm{H} 1299$ parental or CD133-negative cells (Fig. 2A). Tumor growth curves showed that tumors that had developed from the CD133-positive cells grew at a faster rate compared with those that had developed from the parental or CD133-negative cells (Fig. 2B). These observations indicated that CD133-positive lung cancer cells may be used to enrich tumor-initiating cells under sphere-forming conditions.

Patient information. Tumor specimens from 30 patients (19 paired samples of lung adenocarcinomas and 11 paired samples of lung squamous-cell carcinomas) with previously untreated NSCLC were recruited into the current study. The patients consisted of 23 males and 7 females. The median age 
A

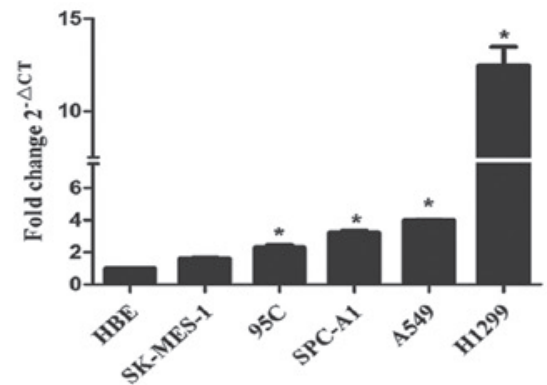

B
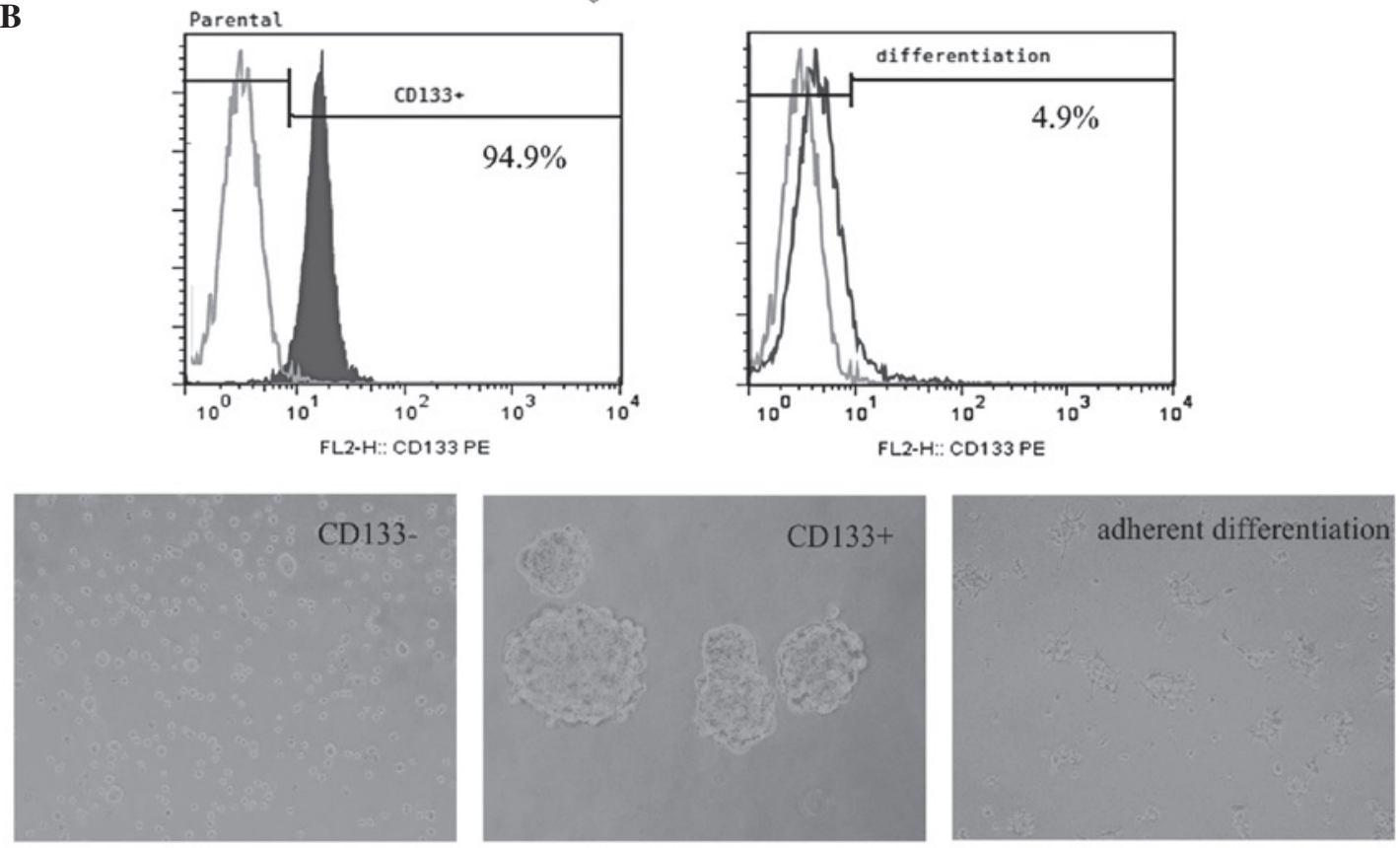

C

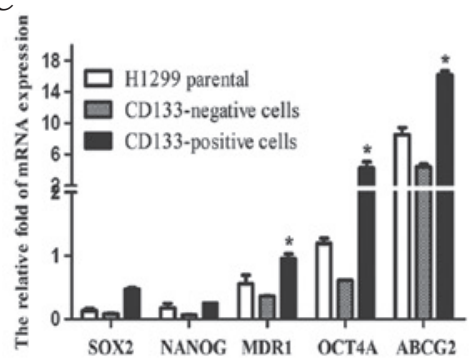

D

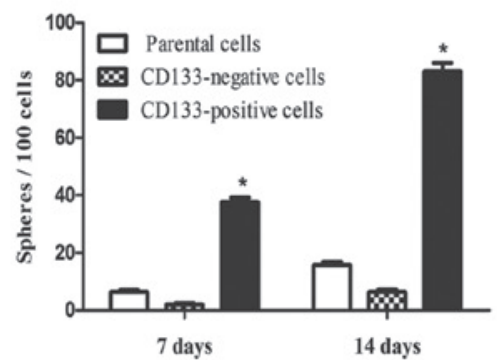

$\mathbf{E}$

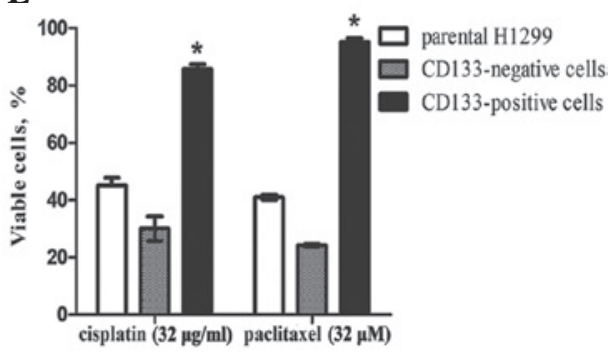

Figure 1. Isolation and characterization of CD133-positive and CD133-negative cells from the H1299 cell line. (A) Expression levels of CD133 were detected by qPCR in a normal bronchial epithelial cell line and a number of non-small cell lung carcinoma cell lines (HBE, SK-MES-1, 95C, SPC-A1, A549 and H1299). The average $\mathrm{Ct}$ value from triplicate assessment in the candidate mRNAs was calculated. ${ }^{*} \mathrm{P}<0.05$, vs. HBE cells. (B) Using a magnetic bead method, CD133-positive cells isolated from H1299 cells were identified by flow cytometry. The image shows spheroid-like bodies formed by CD133-positive and CD133-negative cells in serum-free medium containing bFGF and EGF. The differentiated cells, which were identified to be CD133-positive cells, were grown in adherent conditions in differentiating culture medium for 4 days. Scale bar, $100 \mu \mathrm{m}$. (C) mRNA expression levels of cancer stem cell association genes: OCT4A, SOX2, NANOG, MDR1 and ABCG2 in CD133-positive, CD133-negative and H1299 parental cells were determined by qPCR. The fold changes in the target mRNAs were normalized with that of GAPDH. "P<0.05, vs. CD133-negative and H1299 parental cells. (D) An analysis of the ability of different cell groups to form spheroid-like bodies in the serum-free medium containing bFGF and EGF at the indicated days. "P<0.05, vs. CD133-negative and H1299 parental cells. (E) Effect of cisplatin and paclitaxel on the proliferation of parental H1299, CD133-positive or CD133-negative cells. The cells (5x103) were plated in a 96-well plate and treated with the indicated concentrations of cisplatin and paclitaxel for $24 \mathrm{~h}$. The survival rate was determined by a cell counting kit-8 assay. Data are presented as the mean \pm SD of three independent experiments. " $\mathrm{P}<0.05$, vs. CD133-negative and H1299 parental cells. qPCR, quantitative polymerase chain reaction; $\mathrm{Ct}$, cycle threshold; bFGF, basic fibroblast growth factor; EGF. epidermal growth factor; OCT4A, octamer-binding transcription factor 4; SOX2, sex determining region Y-box 2; NANOG, Nanog homeobox; MDR1, multidrug resistance protein 1; ABCG2, ATP-binding cassette subfamily G member 2; GAPDH, glyceraldehyde 3-phosphate dehydrogenase.

was 61.1 years, with nine cases $(30 \%)$ aged $>65$ years. Based on the World Health Organization criteria, 15 patients $(50 \%)$ presented with stage I disease, six patients $(20 \%)$ with stage II disease and 9 patients $(30 \%)$ with stage III/IV disease. Of the 30 patients, $14(46.67 \%)$ had tumors with nodal metastasis and $15(30 \%)$ had a primary tumor $>3 \mathrm{~cm}$ in diameter. The majority of the patients $(22 ; 73.33 \%)$ were smokers.

Cancer stem cell-associated gene expression in lung tissue. A primary aim of the current study was to determine whether 
A

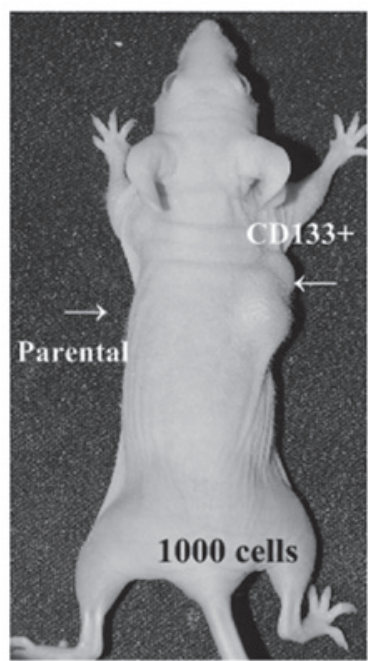

B

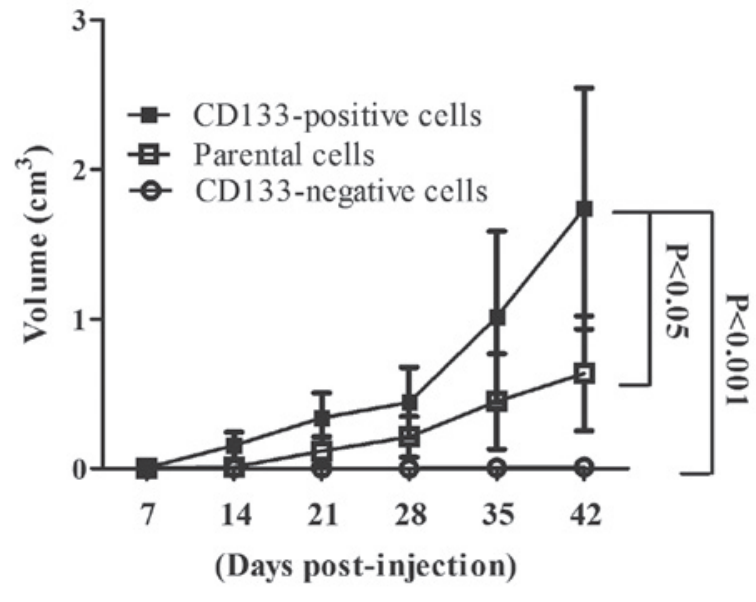

Figure 2. Tumorigenic potential of CD133-positive cells in vivo. (A) Cells ( $10^{3}$ cells in the CD133-positive cell and parental cell group) were simultaneously injected into the right and left flank of the same mouse and the image was captured 2 months following tumor cell implantation. The image represents the relative tumorigenic potential of the CD133-positive cells compared with parental cells in vivo. The arrows indicate the injected tumor formation. (B) Tumor growth curves were generated from isolated CD133-positive, CD133-negative and H1299 parental cells ( $10^{6}$ cells in each group). Cells were subcutaneously injected into nude mice and tumor growth was monitored over time ( $n=4) .{ }^{*} \mathrm{P}<0.05$, vs. H1299 parental cells.

A

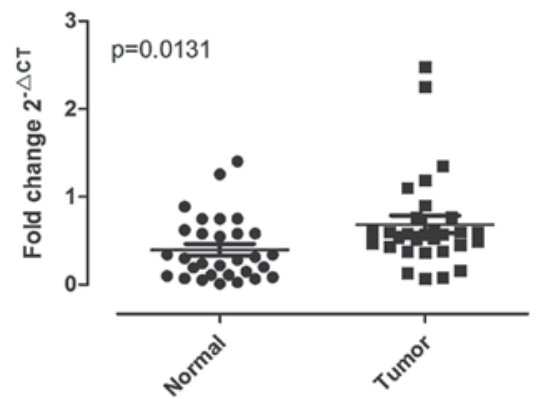

C

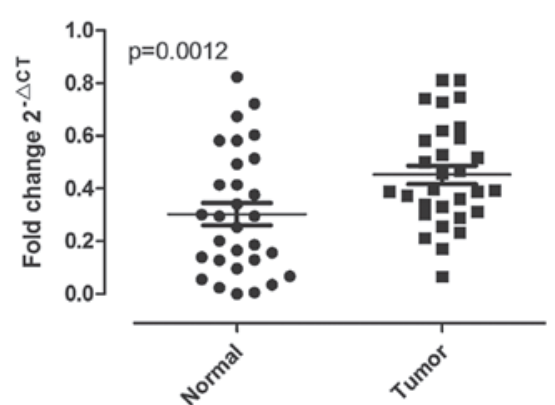

B

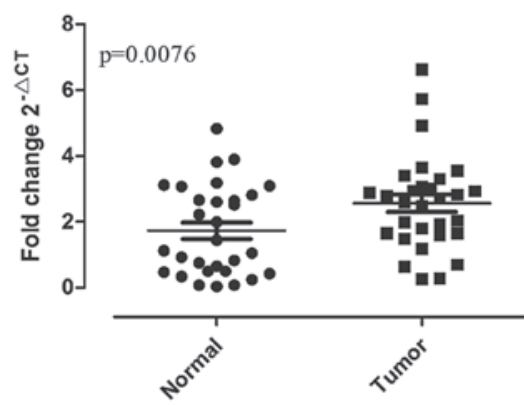

D

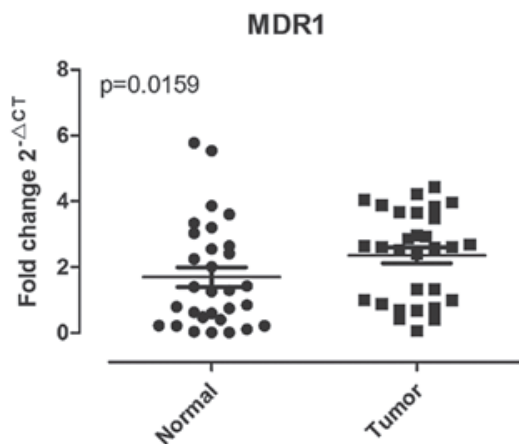

Figure 3. CSC-associated gene expression in lung tissue. Total RNA was isolated from the 30 paired tumors and normal lung tissue and qPCR was performed to detect CSC-associated gene expression of (A) CD133, (B) OCT4A, (C) NANOG and (D) MDR1. Expression of mRNA levels were measured using the 2- ${ }^{\Delta 4}$ method. CSC, cancer stem cell; OCT4A, octamer-binding transcription factor 4; NANOG, nanog homeobox; MDR1, multidrug resistance protein 1.

there was a difference in the frequency of CSC-associated gene expression between paired lung carcinoma and the corresponding noncancerous lung tissue. The results from the $\mathrm{qPCR}$ analysis showed that, compared with the adjacent normal lung tissues, the levels of CSC-associated biomarkers, such as OCT4A, CD133, NANOG and MDR1, were significantly increased in lung cancer tissues $(\mathrm{P}<0.05$; Fig. 3$)$. The mRNA levels of CD133 (Fig. 3A) and MDR1 (Fig. 3D) were observed to be significantly higher in the tumor tissue (CD133, $\mathrm{P}=0.0131$ and MDR 1, $\mathrm{P}=0.0159$ ) compared with that in adjacent normal lung tissue. In addition, compared with the normal counterpart, higher NANOG and OCT4A expression was observed in the malignant tissue $(\mathrm{P}=0.0012$ and $\mathrm{P}=0.0076)$ in 23 and 19 of 30 pairs (76.67 and 63.33\%, respectively; Fig. 3C and B).

Correlation between the expression of cancer stem cell-associated genes and clinical-pathological features of NSCLC. The correlation between the expression of CSC-associated genes 
Table III. Correlation between the expression of CSC-associated genes and the clinicopathological factors in patients with NSCLC.

\begin{tabular}{|c|c|c|c|c|c|c|c|c|c|}
\hline & & OCT4A & & NANOG & & MDR1 & & CD133 & \\
\hline Variables & $\mathrm{n}=30$ & Mean \pm SEM & P-value & Mean \pm SEM & P-value & Mean \pm SEM & P-value & Mean \pm SEM & P-value \\
\hline Gender & & & 0.1285 & & $0.0470^{\mathrm{a}}$ & & 0.0558 & & 0.1224 \\
\hline Male & 23 & $6.049 \pm 2.184$ & & $9.195 \pm 4.362$ & & $11.75 \pm 8.49$ & & $5.821 \pm 1.829$ & \\
\hline Female & 7 & $1.081 \pm 0.1406$ & & $1.461 \pm 0.3930$ & & $1.310 \pm 0.3004$ & & $1.235 \pm 0.2130$ & \\
\hline Age (years) & & & 0.4385 & & $0.0388^{\mathrm{a}}$ & & 0.2452 & & 0.2917 \\
\hline$\leq 60$ & 11 & $5.485 \pm 4.112$ & & $6.080 \pm 4.851$ & & $19.26 \pm 17.88$ & & $4.816 \pm 3.389$ & \\
\hline$>60$ & 19 & $4.546 \pm 1.412$ & & $8.149 \pm 4.632$ & & $3.550 \pm 0.6745$ & & $4.713 \pm 1.262$ & \\
\hline Histological type & & & $0.0034^{\mathrm{a}}$ & & 0.6498 & & 0.1138 & & $0.0024^{\mathrm{a}}$ \\
\hline Squamous carcinoma & 11 & $11.17 \pm 4.099$ & & $9.460 \pm 4.631$ & & $22.93 \pm 17.53$ & & $10.19 \pm 3.336$ & \\
\hline Adenocarcinoma & 19 & $1.256 \pm 0.2140$ & & $6.192 \pm 4.690$ & & $1.429 \pm 0.2178$ & & $1.604 \pm 0.4545$ & \\
\hline Pathological stage & & & $0.0417^{\mathrm{a}}$ & & 0.1539 & & 0.1132 & & $0.0334^{\mathrm{a}}$ \\
\hline $\mathrm{I}, \mathrm{II}$ & 21 & $6.009 \pm 2.272$ & & $9.769 \pm 4.767$ & & $12.47 \pm 9.301$ & & $5.800 \pm 1.976$ & \\
\hline III, IV & 9 & $2.280 \pm 1.125$ & & $1.841 \pm 0.4646$ & & $1.944 \pm 0.608$ & & $2.302 \pm 1.125$ & \\
\hline Smoking status & & & 0.0710 & & 0.0516 & & $0.0292^{\mathrm{a}}$ & & 0.0827 \\
\hline Nonsmokers & 8 & $1.046 \pm 0.1265$ & & $1.472 \pm 0.3405$ & & $1.264 \pm 0.2641$ & & $1.201 \pm 0.1875$ & \\
\hline Current smokers & 22 & $6.288 \pm 2.272$ & & $9.542 \pm 4.551$ & & $12.24 \pm 8.870$ & & $6.041 \pm 1.900$ & \\
\hline Tumor size $(\mathrm{cm})$ & & & 0.0680 & & 0.0970 & & 0.1776 & & $0.0238^{\mathrm{a}}$ \\
\hline$<3$ & 15 & $1.838 \pm 0.5074$ & & $2.435 \pm 0.8169$ & & $2.090 \pm 0.554$ & & $1.865 \pm 0.5416$ & \\
\hline$\geq 3$ & 15 & $7.942 \pm 3.247$ & & $12.35 \pm 6.576$ & & $16.53 \pm 12.99$ & & $7.636 \pm 2.669$ & \\
\hline Grade of differentiation & & & $0.0396^{\mathrm{a}}$ & & $0.0142^{\mathrm{a}}$ & & $0.0248^{\mathrm{a}}$ & & $0.0376^{\mathrm{a}}$ \\
\hline Moderate-well & 16 & $1.611 \pm 0.4888$ & & $2.257 \pm 0.8798$ & & $1.877 \pm 0.5741$ & & $1.761 \pm 0.5459$ & \\
\hline Poor & 14 & $7.759 \pm 3.043$ & & $11.88 \pm 6.166$ & & $15.82 \pm 12.17$ & & $7.366 \pm 2.516$ & \\
\hline Lymph node status & & & 0.1904 & & 0.3940 & & 0.3237 & & 0.2530 \\
\hline No & 16 & $5.645 \pm 2.796$ & & $11.64 \pm 6.213$ & & $15.14 \pm 12.21$ & & $5.565 \pm 2.329$ & \\
\hline $\mathrm{N}^{+}$ & 14 & $4.028 \pm 1.892$ & & $2.535 \pm 0.6427$ & & $2.782 \pm 0.8021$ & & $3.820 \pm 1.629$ & \\
\hline
\end{tabular}

${ }^{a} \mathrm{P}<0.05$ indicates a statistically significant difference. NSCLC, non-small cell lung carcinoma; CSC, cancer stem cell; OCT4A, octamer-binding transcription factor 4; NANOG, Nanog homeobox; MDR1, multidrug resistance protein 1.

and clinicopathological features of NSCLC was investigated to gain an improved understanding of the potential involvement of these genes in NSCLC development and progression using the Mann-Whitney U test (Table III). No substantial correlation between the expression of NANOG or MDR1 and the tumor stage, nodal status, tumor size and histological type was observed, with the exception of tumor differentiation. However, overexpression of CD133 in lung tumors was observed to be markedly associated with clinical stage, tumor size and differentiation of NSCLC $(\mathrm{P}=0.0334,0.0238$ and 0.0376, respectively; Fig. 4A-C). In addition, patients with squamous-cell lung carcinoma or a tumor $\geq 3 \mathrm{~cm}$ in diameter exhibited higher expression levels of CD133. Notably, overexpression of OCT4A in tumors was positively associated with a tumor stage I or II and moderate-well differentiated tumors $(\mathrm{P}=0.0417$ and 0.0396 , respectively; Fig. 4D and $\mathrm{E})$. In addition, patients with poor differentiation had higher levels of CD133 and OCT4A.

Correlation between cancer stem cell-associated gene expression and overall survival of NSCLC patients. The expression of CSC-associated genes and its correlation with overall survival of NSCLC patients was investigated (fold change
$>2$ was considered as high expression). The results showed that higher levels of expression of CD133 in NSCLC was independently correlated with shorter overall survival (log-rank test: $\mathrm{P}=0.0258$, Fig. 5). However, no significant correlation between the expression of OCT4A and the overall survival was observed (log-rank test: $\mathrm{P}=0.1395)$. In addition, tumor size $>3 \mathrm{~cm}$ was observed to be associated with decreased overall survival (log-rank test: $\mathrm{P}=0.015$, data not shown).

The Kaplan-Meier survival curve showed a trend for an improved outcome in patients with lower CD133 mRNA signals; however, the Cox hazard regression analysis indicated that CD133 was not considered to be an independent factor in predicting the prognosis of patients with NSCLC (hazard ratio: $0.179 ; 95 \%$ confidence interval: $0.010-3.205 ; \mathrm{P}=0.243$ ).

\section{Discussion}

Lung cancer is characterized by the difficulty surrounding early diagnosis, high rates of metastasis, recurrence and poor prognosis. Thus, investigating novel diagnostic strategies and novel prognostic markers is urgently required to improve the clinical outcome of this malignancy (1). Increased numbers of CD133-positive cancer stem cells have been observed in 
A

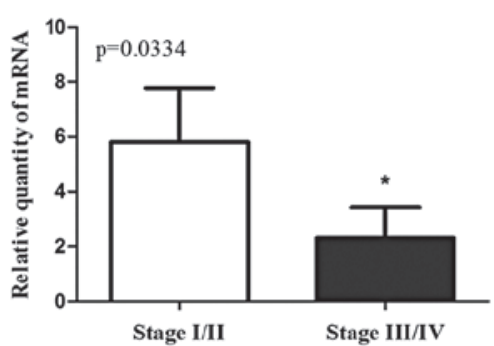

B

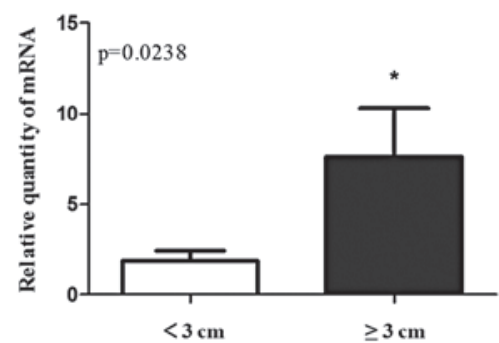

C

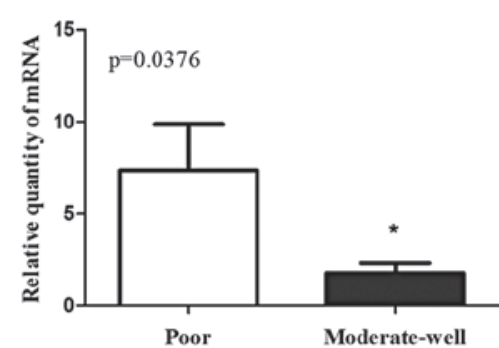

D

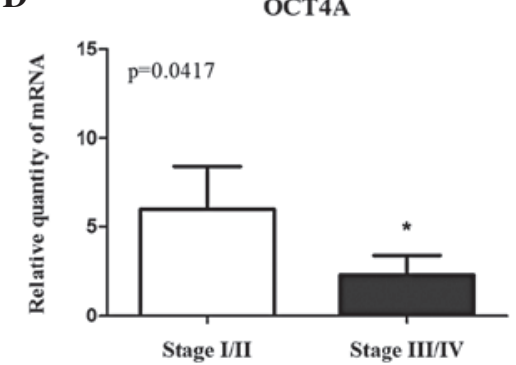

$\mathbf{E}$

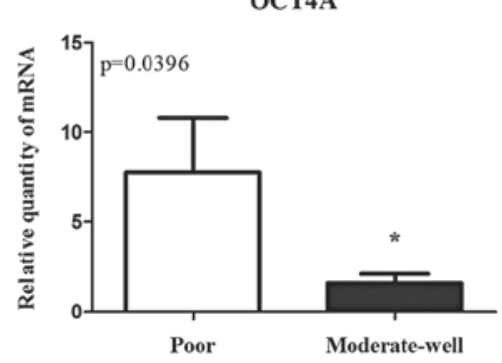

Figure 4. Correlation between the expression of CSC-associated genes and clinicopathological features of NSCLC. The correlation between the expression of mRNA levels of relevant CSC-associated genes and clinical-pathological features of the patients with NSCLC were analyzed by the Mann-Whitney U test. The association of CD133 with (A) stage, (B) tumor size and (C) tumor differentiation and the OCT4A correlation with (D) stage and (E) tumor differentiation were determined. ${ }^{*} \mathrm{P}<0.05$, vs. two groups. CSC, cancer stem cell; NSCLC, non-small cell lung carcinoma; OCT4A, octamer-binding transcription factor 4.

NSCLC (13). In the current study, isolated CD133-positive cells were shown to harbor stem cell-like characteristics, as they readily form anchorage-independent floating spheres, possess greater proliferative potential and exhibit enhanced tumor regenerating capacity compared with their CD133-negative counterparts. In addition, CD133-positive, but not CD133-negative or parental NSCLC cells formed tumors in the nude mice xenograft model. These results demonstrated the CSC status of CD133-positive cells in NSCLC.

The correlation of CSC-associated gene expression (CD133, OCT4A, NANOG and MDR1) in NSCLC samples, with patient characteristics, tumor pathology and overall survival was investigated. The current results indicated the presence of CSCs in NSCLC tissue, as self-renewal and multipotency are critical characteristics of CSCs (4). Based on the present observations, the correlation between increased CD133 expression and early stage tumors, larger tumor size and poorly differentiated NSCLC tumors may be to be due to the self-renewal properties of aberrant CSCs. This implicated the importance of CD133-positive cells in the initiation of NSCLC. The reduced levels of CD133 in the later stages of NSCLC may be explained by the hypothesis that cells expressing CD133 undergo asymmetric division, generating a diverse phenotype and therefore resulting in reduced CD133 positivity. However, further studies are required to confirm this.

Previous studies have shown that the expression of CSC antigens was associated with a poor prognosis $(20-22,25,26)$. However, the current observations, which are consistent with the results of other studies (17-19), demonstrated that while the expression of CD133 in the surgically resected specimens was involved in NSCLC carcinogenesis, CD133 alone may not be used as an independent biomarker in the prediction of the prognosis of NSCLC. The origin of these discrepancies

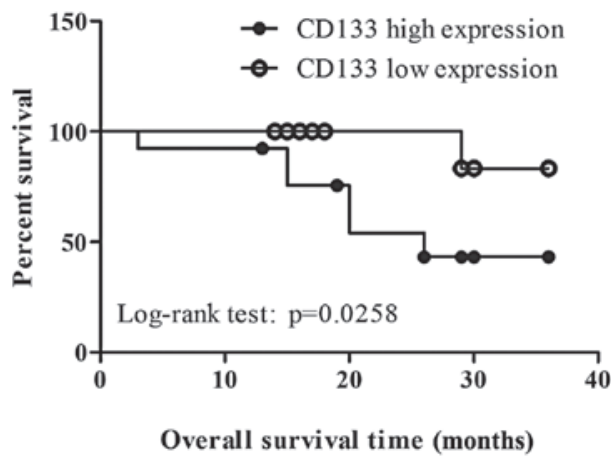

Figure 5. The Kaplan-Meier method and the log-rank test were used to compare the overall survival in high and low CD133 expression groups. Kaplan-Meier survival curves for NSCLC patients were plotted for expression, which was based on CD133 cut-off values. The P-value was calculated using the log-rank test between patients with high- and low-fold changes. The overall survival of patients with high vs. low expression levels of CD133 is shown. $\mathrm{P}<0.05$, vs. the two groups. CD133, prominin-1; NSCLC, non-small cell lung carcinoma.

remains unclear; however, specific NSCLC samples may be the reason for variability between the results. Notably, the present specimens were obtained from patients with all stages of NSCLC and contained various histological types (adenocarcinoma and squamous carcinoma).

Studies in human embryonic stem cells indicated that OCT4A and the homeobox protein NANOG were two key transcription factors that cooperatively maintained pluripotency $(27,28)$. The present results indicated that OCT4A was significantly associated with the clinical stage and differentiation of NSCLC. Consistent with this, Chen et al (29) observed that knockdown of the OCT4A gene in CD133-positive stem-like lung cancer cells significantly diminished the 
ability of tumor invasion and colony formation, and increased apoptotic activities. The aforementioned observations indicate that the expression of OCT4A was involved in maintaining the stem cell-like properties of lung cancer cells. Whether the combination of the two CSC markers, OCT4 and CD133, may enhance the predictive validity of patient progression and survival remains to be elucidated.

In conclusion, the results of the present study show that CD133-positive NSCLC cells exhibit clonogenic, tumorigenic and drug-resistant properties. The elevated expression of CD133 was associated with early stage tumors, larger tumor size and poor differentiation of NSCLC, indicating that highly expressed CD133 is involved in the carcinogenesis of NSCLC. However, expression of CD133 in CSCs is not considered to be an independent factor in the prediction of the prognosis of patients with NSCLC. Further studies are required to determine the association between CD133 expression and overall survival in NSCLC patients, with or without other CSC-associated genes.

\section{Acknowledgements}

This study was supported by grants from the Zhejiang Provincial Natural Science Foundation of China (grant no. Y2101395) and the Medical Bureau of Zhoushan (grant no. 2010G02).

\section{References}

1. Spira A and Ettinger DS: Multidisciplinary management of lung cancer. N Engl J Med 350: 379-392, 2004.

2. Reya T, Morrison SJ, Clarke MF and Weissman IL: Stem cells, cancer, and cancer stem cells. Nature 414: 105-111, 2001.

3. van Klaveren RJ, van't Westeinde SC, de Hoop BJ and Hoogsteden HC: Stem cells and the natural history of lung cancer: implications for lung cancer screening. Clin Cancer Res 15: 2215-2218, 2009

4. Sullivan JP, Minna JD and Shay JW: Evidence for self-renewing lung cancer stem cells and their implications in tumor initiation, progression, and targeted therapy. Cancer Metastasis Rev 29: 61-72, 2010.

5. Kim CF, Jackson EL, Woolfenden AE, Lawrence S, Babar I, Vogel S, Crowley D, Bronson RT and Jacks T: Identification of bronchioalveolar stem cells in normal lung and lung cancer. Cell 121: 823-835, 2005.

6. Rosen JM and Jordan CT: The increasing complexity of the cancer stem cell paradigm. Science 324: 1670-1613, 2009

7. Singh SK, Hawkins C, Clarke ID, Squire JA, Bayani J, Hide T, Henkelman RM, Cusimano MD and Dirks PB: Identification of human brain tumour initiating cells. Nature 432: 396-401, 2004.

8. Zhao P, Lu Y, Jiang X and Li X: Clinicopathological significance and prognostic value of CD133 expression in triple-negative breast carcinoma. Cancer Sci 102: 1107-1111, 2011.

9. Yin S, Li J, Hu C, Chen X, Yao M, Yan M, Jiang G, Ge C, Xie H, Wan D, Yang S, Zheng S and Gu J: CD133 positive hepatocellular carcinoma cells possess high capacity for tumorigenicity. Int J Cancer 120: 1444-1450, 2007.

10. Hermann PC, Huber SL, Herrler T, Aicher A, Ellwart JW, Guba M, Bruns CJ and Heeschen C: Distinct populations of cancer stem cells determine tumor growth and metastatic activity in human pancreatic cancer. Cell Stem Cell 1: 313-323, 2007.

11. Ricci-Vitiani L, Lombardi DG, Pilozzi E, Biffoni M, Todaro M, Peschle $C$ and De Maria R: Identification and expansion of human colon-cancer-initiating cells. Nature 445: 111-115, 2007.
12. Giangreco A, Groot KR and Janes SM: Lung cancer and lung stem cells: strange bedfellows? Am J Respir Crit Care Med 175: 547-553, 2007.

13. Eramo A, Lotti F, Sette G, Pilozzi E, Biffoni M, Di Virgilio A, Conticello C, Ruco L, Peschle C and De Maria R: Identification and expansion of the tumorigenic lung cancer stem cell population. Cell Death Differ 15: 504-514, 2008.

14. Meng X, Li M, Wang X, Wang Y and Ma D: Both CD133+ and CD133- subpopulations of A549 and H446 cells contain cancer-initiating cells. Cancer Sci 100: 1040-1046, 2009.

15. Qiu X, Wang Z, Li Y, Miao Y, Ren Y and Luan Y: Characterization of sphere-forming cells with stem-like properties from the small cell lung cancer cell line H446. Cancer Lett 323: 161-170, 2012.

16. Akunuru S, James Zhai Q and Zheng Y: Non-small cell lung cancer stem/progenitor cells are enriched in multiple distinct phenotypic subpopulations and exhibit plasticity. Cell Death Dis 3: e352, 2012.

17. Salnikov AV, Gladkich J, Moldenhauer G, Volm M, Mattern J and Herr I: CD133 is indicative for a resistance phenotype but does not represent a prognostic marker for survival of non-small cell lung cancer patients. Int J Cancer 126: 950-958, 2010.

18. Herpel E, Jensen K, Muley T, Warth A, Schnabel PA, Meister M, Herth FJ, Dienemann H, Thomas M and Gottschling S: The cancer stem cell antigens CD133, BCRP1/ABCG2 and CD117/c-KIT are not associated with prognosis in resected early-stage non-small cell lung cancer. Anticancer Res 31: 4491-4500, 2011.

19. Shien K, Toyooka S, Ichimura K, Soh J, Furukawa M, Maki Y, Muraoka T, Tanaka N, Ueno T, Asano H, et al: Prognostic impact of cancer stem cell-related markers in non-small cell lung cancer patients treated with induction chemoradiotherapy. Lung Cancer 77: 162-167, 2012.

20. Cortes-Dericks L, Galetta D, Spaggiari L, Schmid RA and Karoubi G: High expression of octamer-binding transcription factor 4A, prominin-1 and aldehyde dehydrogenase strongly indicates involvement in the initiation of lung adenocarcinoma resulting in shorter disease-free intervals. Eur J Cardiothorac Surg 41: e173-e181, 2012.

21. Woo T, Okudela K, Mitsui H, Yazawa T, Ogawa N, Tajiri M, Yamamoto T, Rino Y, Kitamura H and Masuda M: Prognostic value of CD133 expression in stage I lung adenocarcinomas. Int J Clin Exp Pathol 4: 32-42, 2010.

22. Li F, Zeng H and Ying K: The combination of stem cell markers CD133 and ABCG2 predicts relapse in stage I non-small cell lung carcinomas. Med Oncol 28: 1458-1462, 2011.

23. Ettinger DS, Akerley W, Borghaei H, et al: Non-Small Cell Lung Cancer, Version 2.2013. J Natl Compr Canc Netw 11: 645-653, 2013.

24. Livak KJ and Schmittgen TD: Analysis of relative gene expression data using real-time quantitative PCR and the 2(-Delta Delta C(T)) Method. Methods 25: 402-408, 2001.

25. Zeppernick F, Ahmadi R, Campos B, Dictus C, Helmke BM, Becker N, Lichter P, Unterberg A, Radlwimmer B and Herold-Mende CC: Stem cell marker CD133 affects clinical outcome in glioma patients. Clin Cancer Res 14: 123-129, 2008.

26. Sullivan JP, Spinola M, Dodge M, Raso MG, Behrens C, Gao B, Schuster K, Shao C, Larsen JE, Sullivan LA, et al: Aldehyde dehydrogenase activity selects for lung adenocarcinoma stem cells dependent on notch signaling. Cancer Res 70: 9937-9948, 2010.

27. Nichols J,Zevnik B, Anastassiadis K, Niwa H, Klewe-Nebenius D, Chambers I, Schöler H and Smith A: Formation of pluripotent stem cells in the mammalian embryo depends on the POU transcription factor Oct4. Cell 95: 379-391, 1998

28. Chiou SH, Yu CC, Huang CY, Lin SC, Liu CJ, Tsai TH, Chou SH, Chien CS, Ku HH and Lo JF: Positive correlations of Oct-4 and Nanog in oral cancer stem-like cells and high-grade oral squamous cell carcinoma. Clin Cancer Res 14: 4085-4095, 2008.

29. Chen YC, Hsu HS, Chen YW, Tsai TH, How CK, Wang CY, Hung SC, Chang YL, Tsai ML, Lee YY, Ku HH and Chiou SH: Oct-4 expression maintained cancer stem-like properties in lung cancer-derived CD133-positive cells. PLoS One 3: e2637, 2008. 